\title{
ANGPTL2 is associated with an increased risk of cardiovascular events and death in diabetic patients
}

\author{
Barnabas Gellen $^{1,2,3}$ (D) Nathalie Thorin-Trescases ${ }^{4} \cdot$ Philippe Sosner $^{5,6,7} \cdot$ Elise Gand $^{8}$. \\ Pierre-Jean Saulnier ${ }^{5,9,10}$ - Stéphanie Ragot $^{5}$ • Mathilde Fraty ${ }^{8}$ - Stéphanie Laugier ${ }^{10,11}$. \\ Grégory Ducrocq ${ }^{12,13,14}$ • David Montaigne ${ }^{15,16,17,18,19} \cdot$ Pierre Llaty $^{5}$. \\ Vincent Rigalleau $^{20}$ • Philippe Zaoui ${ }^{21,22}$ • Jean-Michel Halimi ${ }^{23}$ - Ronan Roussel ${ }^{12,13}$. $^{2}$ \\ Eric Thorin ${ }^{4}$ • Samy Hadjadj ${ }^{5,9,10,11,24}$
}

Received: 22 April 2016/Accepted: 12 July 2016/Published online: 4 August 2016

(C) Springer-Verlag Berlin Heidelberg 2016

\begin{abstract}
Aims/hypothesis A high serum angiopoietin-like 2 (ANGPTL2) concentration is an independent risk factor for developing diabetes and is associated with insulin resistance and atherosclerosis. In this work, we have examined the impact of serum ANGPTL2 on improving cardiovascular (CV) risk stratification in patients with type 2 diabetes.
\end{abstract}

Eric Thorin and Samy Hadjadj contributed equally to this study.

Electronic supplementary material The online version of this article (doi:10.1007/s00125-016-4066-5) contains peer-reviewed but unedited supplementary material, which is available to authorised users.

Barnabas Gellen

barnabas.gellen.cardio@gmail.com

1 ELSAN, Polyclinique de Poitiers, 1 Rue de la Providence, F-8600 Poitiers, France

2 Department of Cardiology, CHU Henri Mondor, Créteil, France

3 Faculté de Médecine et Pharmacie, Université de Poitiers, Poitiers, France

4 Department of Surgery, Faculty of Medicine, Université de Montréal, Montreal Heart Institute, Montreal, QC, Canada

5 CHU de Poitiers Centre d'Investigation Clinique, Poitiers, France

6 Université de Poitiers, Laboratoire MOVE (EA 6314), Poitiers, France

7 Centre Médico-Sportif Mon Stade, Paris, France

8 CHU de Poitiers, Pôle Dune, Poitiers, France

9 Inserm, CIC 1402, Poitiers, France

10 UFR Médecine Pharmacie, Université de Poitiers, Poitiers, France

11 Endocrinologie - Diabétologie, CHU de Poitiers, Poitiers, France
Methods A prospective, monocentric cohort of consecutive type 2 diabetes patients (the SURDIAGENE cohort; total of 1353 type 2 diabetes patients; $58 \%$ men, mean \pm SD age 64 \pm 11 years) was followed for a median of 6.0 years for death as primary endpoint and major adverse $\mathrm{CV}$ events (MACE; i.e. $\mathrm{CV}$ death, myocardial infarction or stroke) as a secondary endpoint. Patients with end-stage renal disease, defined as a requirement for dialysis or a history of kidney transplantation,

12 Département Hospitalo-Universitaire FIRE, APHP, Hôpital Bichat, Paris, France

13 Faculté de Médecine, Université Paris Diderot, Sorbonne Paris Cité, Paris, France

14 Inserm U-1148, Paris, France

15 CHU Lille, Service d'Explorations Fonctionnelles Cardiovasculaires, Hôpital Cardiologique, Lille, France

16 Faculté de Médecine, Université de Lille, Lille, France

17 Inserm U1011, Lille, France

18 EGID, Lille, France

19 Institut Pasteur de Lille, Lille, France

20 CHU Bordeaux, Haut-Lévêque Hospital, Nutrition-Diabetology Department, Pessac, France

21 CHU de Grenoble, Service Néphrologie, Dialyse et Transplantation, Grenoble, France

22 Faculté de Médecine, Université Joseph Fournier, Grenoble, France

23 CHU de Tours, Service Néphrologie, Dialyse et Transplantation, Tours, France

24 Inserm U1082, Poitiers, France 
were excluded. Patients were grouped into quartiles according to ANGPTL2 concentrations at inclusion: $<11.2(\mathrm{Q} 1), 11.2-$ 14.7 (Q2), 14.8-19.5 (Q3) or >19.5 (Q4) ng/ml.

Results During follow up, 367 patients (representing $4.5 \%$ of the total person-years) died and 290 patients (representing $3.7 \%$ of the total person-years) presented with MACE. Both the survival and MACE-free survival rates were significantly different between ANGPTL2 quartiles (logrank 82.12, $p<0.0001$ for death; and logrank 65.14, $p<0.0001$ for MACE). Patients with ANGPTL2 concentrations higher than $19.5 \mathrm{ng} / \mathrm{ml}(\mathrm{Q} 4)$ had a significantly higher risk of death and MACE than those with ANGPTL2 levels of $19.5 \mathrm{ng} / \mathrm{ml}$ or less (Q1-3) (HR for death 2.44 [95\% CI 1.98, 3.00], $p<0.0001$; HR for MACE 2.43 [95\% CI 1.92, 3.06], $p<0.0001$ ) after adjustment for sex, age and established CV risk factors. Using ANGPTL2 concentrations, prediction of the risk of mortality, as assessed by integrated discrimination improvement (IDI), was significantly improved (IDI $0.006 \pm 0.002$, $p=0.0002$ ).

Conclusions/interpretation In patients with type 2 diabetes, serum ANGPTL2 concentrations were independently associated with death and MACE. Therefore, ANGPTL2 is a promising candidate biomarker for improving risk stratification in type 2 diabetes patients, and may prove to be a valuable therapeutic target.

Keywords Angiopoietin-like 2 - ANGPTL2 - Biomarker . Death $\cdot$ MACE $\cdot$ Type 2 diabetes

$\begin{array}{ll}\text { Abbreviations } \\ \text { ANGPTL2 } & \text { Angiopoietin-like 2 protein } \\ \text { CV } & \text { Cardiovascular } \\ \text { CVD } & \text { Cardiovascular disease } \\ \text { IDI } & \text { Integrated discrimination improvement } \\ \text { MACE } & \text { Major adverse cardiovascular events } \\ \text { NT-proBNP } & \text { N-terminal pro-B-type natriuretic peptide } \\ \text { TNFR1 } & \text { TNF receptor 1 }\end{array}$

\section{Introduction}

Cardiovascular (CV) disease (CVD) constitutes the major determinant of outcomes in patients with type 2 diabetes mellitus in terms of mortality and morbidity [1]. As compared with individuals without diabetes, type 2 diabetes mellitus is associated with a twofold increased risk of mortality and a threefold increased risk of coronary artery disease [2]. CVD risk stratification in type 2 diabetes patients is based on the one hand on the evaluation of classical CV risk factors such as LDL-cholesterol concentrations and smoking, and on the other hand on diabetes-related variables such as $\mathrm{HbA}_{1 \mathrm{c}}$ [3-5].
In addition to established risk factors, several biomarkers associated with atherosclerosis, inflammation and congestive heart failure [6-11] have recently been proposed to improve risk stratification in type 2 diabetes mellitus patients. Promising data have been obtained using biomarkers associated with the closely interlinked processes of atherosclerosis and low-grade systemic inflammation [12]. However, none of these biomarkers has been introduced into online riskstratification engines or international guidelines.

Angiopoietin-like 2 (ANGPTL2) is a proinflammatory circulating protein [13] that has been related to chronic inflammatory diseases such as atherosclerosis $[14,15]$, diabetes [15, 16], cancer [17] and many others [18]. It is produced by endothelial cells [14, 15], adipocytes [15, 19] and macrophages [20]. Abundantly expressed in visceral fat [15], ANGPTL2 is involved in chronic low-grade inflammation, and might contribute to obesity-related systemic insulin resistance $[15,21]$. In healthy individuals, increased serum ANGPTL2 concentrations have been found to be associated with a higher risk of developing type 2 diabetes mellitus [16]. Furthermore, patients with heart failure have been found to have increased ANGPTL2 concentrations, as compared with a healthy reference population [22]. Recently, higher ANGPTL2 levels have also been observed in patients with acute coronary syndrome [23]. These findings suggest a link between serum ANGPTL2 concentrations and CV risk factors and $\mathrm{CV}$ disease, and it has recently been proposed that ANGPTL2 may be a promising therapeutic target [18].

In the present study, we examined whether measurement of serum ANGPTL2, in addition to established risk markers, is useful in the long-term risk assessment of type 2 diabetes mellitus patients with regard to all-cause mortality and CV morbidity and mortality.

\section{Methods}

Patients were prospectively included in this single-centre cohort (the 'SURDIAGENE' cohort) from 2002 to 2011 and followed until 2013 [24]. The study design was approved by the local ethics committee, and written informed consent was obtained from all participants.

Inclusion and exclusion criteria Adult patients with type 2 diabetes mellitus were consecutively recruited and followed regularly at the University Hospital of Poitiers, France. The main exclusion criteria were residence outside of the Poitiers region and evidence of non-diabetic renal disease. For this analysis, additional exclusion criteria were the presence at study admission of a stage 4 or worse chronic kidney disease (GFR $<30 \mathrm{ml} \mathrm{min}^{-1}[1.73 \mathrm{~m}]^{-2}$; renal replacement therapy) and a follow-up duration of less than 3 months. 
Demographic, clinical and biological variables Clinical data were obtained at inclusion from patient records, and morphometric measurements, BP and ECG recordings were taken at inclusion. A self-reported history of myocardial infarction or symptomatic peripheral artery disease was noted. Biological data were also collected at inclusion. $\mathrm{HbA}_{1 \mathrm{c}}$ and serum creatinine concentrations were determined centrally in the fasting state using a chromatography method (ADAMS $\mathrm{A}_{1 \mathrm{c}}$ HA-8160 analyser; Menarini, Florence, Italy) and a colorimetric method on an automated analyser (KONE Optima; Thermo Clinical Labsystems, Vantaa, Finland), respectively. The eGFR was calculated using the Chronic Kidney Disease Epidemiology Collaboration formula [25]. Urinary creatinine was measured on a Hitachi 911 automatic analyser (Roche Diagnostics, Meylan, France), and urinary albumin by nephelometry on a Modular System P (Roche Diagnostics).

Serum concentrations of TNF receptor 1 (TNFR1), a marker of inflammation, were measured using a human soluble TNFR1 ELISA kit (EKF Diagnostics, Dublin, Ireland). All serum samples were tested in duplicate, and the mean of the two measurements was taken for statistical analysis. Serum ANGPTL2 concentrations were measured using a human ANGPTL2 ELISA kit (Cloud-Clone Corp., Houston, TX, USA) according to the manufacturer's instructions. Serum samples were diluted (1:2, or 1:4 if needed) using the kit standard diluent solution. A serum sample from one healthy volunteer was always included on each ELISA plate in order to validate the assay; inter- and intra-assay variability were $<15 \%$ and $<10 \%$, respectively. Optical densities (450 nm) of two standard curves were averaged and a model of non-linear regression with four variables was used to calculate ANGPTL2 concentrations. This model allowed for the calculation of ANGPTL2 concentrations up to $60 \mathrm{ng} / \mathrm{ml}$ in samples diluted 1:2; above this threshold, samples were re-taken and diluted 1:4.

Study outcomes Whether each patient was alive or dead and CV endpoints were determined from patients' hospital records, interviews with their general practitioners and inquiry to the French National Death Registry. The present analysis takes the most recent available data (2013) into account. The primary endpoint was all-cause mortality. The secondary endpoint was a composite of $\mathrm{CV}$ death, non-fatal myocardial infarction and non-fatal stroke (major adverse CV events [MACE]) [26]. Each event was reviewed by an adjudication committee according to the international definitions of clinical outcomes. The adjudication committee was blinded with regard to ANGPTL2 concentrations. We compared the observed risk for all-cause death in our cohort with the risk predicted by the latest version of the UK Prospective Diabetes Study outcome equation using the following variables: age, diabetes duration, sex, ethnicity, current smoking status, systolic $\mathrm{BP}, \mathrm{HbA}_{1 \mathrm{c}}, \mathrm{HDL}$ - and LDL-cholesterol, BMI, eGFR, heart rate, atrial fibrillation, albuminuria and peripheral vascular disease.

Statistical analysis Qualitative variables are reported as absolute values and percentages, while quantitative variables are described by mean \pm SD or median (interquartile range), as appropriate. Associations between qualitative variables were evaluated using the $\chi^{2}$ test. Quantitative variables were analysed using the Student's $t$ test or Mann-Whitney $U$ test, as appropriate.

We considered time to occurrence of the first event as the primary endpoint. Survival curves were built using the Kaplan-Meier method and compared using the logrank test. Risk-prediction models established by Cox proportional hazard models were used to analyse the effect on study outcomes. HRs and their $95 \%$ CIs are presented.

We tested for non-linear associations using cubic splines for ANGPTL2 and non-adjusted risk of all-cause death. Quantitative variables were log-transformed if appropriate in order to meet the application requirement for the Cox model. Variables associated with all-cause death at $p<0.10$ in the univariate Cox model were selected for the multivariate model. The final model was determined using multiple stepwise regression analysis. To assess improvements in the Cox model achieved by adding ANGPTL2, we used the integrated discrimination improvement (IDI) index and Harrell's C index, in addition to established risk markers.

All hypotheses were tested at the 5\% level of significance. Statistical analyses were carried out using the SAS version 9.3 software package (SAS, Cary, NC, USA).

\section{Results}

The median follow-up duration was 72 months (interquartile range 67 months), corresponding to 8143 person-years. The demographic, clinical and biological characteristics of the study population are summarised in Table 1.

The mortality rate was $4.5 \%$ of total person-years $(95 \% \mathrm{CI}$ $4.0,5.0 ; n=367$ ) and the MACE rate was $3.7 \%$ of total person-years $(95 \%$ CI 3.3, 4.1; $n=290)$. The major causes of death were CVD $(n=211 ; 57 \%)$, cancer $(n=56 ; 15 \%)$ and infections $(n=33 ; 9 \%)$.

When compared with the survivor group, patients who died during follow-up had more CV risk factors (including greater impairment of renal function) and also had more prior $\mathrm{CV}$ events (Table 1 ). With regard to type 2 diabetes mellitus, the duration of diabetes was longer and insulin use was more common in those who died, whereas $\mathrm{HbA}_{1 \mathrm{c}}$ levels were comparable between the groups (Table 1).

Serum ANGPTL2 protein concentrations at inclusion were significantly associated with the risk of both death (HR 8.60 [95\% CI 5.36, 13.78]; $p<0.0001$ per $\log \mathrm{ng} / \mathrm{ml})$ and MACE 
Table 1 Baseline patient characteristics according to death

\begin{tabular}{|c|c|c|c|c|}
\hline Variable & All $(n=1353)$ & Event $(n=367)$ & $\begin{array}{l}\text { No event } \\
(n=986)\end{array}$ & $p$ value \\
\hline Men/women, $n(\%)$ & $782(58) / 571(42)$ & $241(66) / 126(34)$ & $541(55) / 445(45)$ & $0.0003^{* * *}$ \\
\hline Age, years & $64 \pm 11$ & $70 \pm 9$ & $63 \pm 11$ & $<0.0001 * * *$ \\
\hline Non-white ethnicity, $n(\%)$ & $43(3)$ & $10(3)$ & $33(3)$ & 0.5620 \\
\hline BMI, $\mathrm{kg} / \mathrm{m}^{2}$ & $31 \pm 6$ & $31 \pm 6$ & $32 \pm 6$ & $0.0107 *$ \\
\hline Active smoking, $n(\%)$ & $148(11)$ & $43(12)$ & $105(11)$ & 0.5738 \\
\hline Hypertension, $n(\%)$ & $1112(82)$ & $329(90)$ & $783(79)$ & $<0.0001 * * *$ \\
\hline $\begin{array}{l}\text { History of myocardial } \\
\text { infarction, } n(\%)\end{array}$ & $200(15)$ & $85(23)$ & $115(12)$ & $<0.0001 * * *$ \\
\hline History of stroke, $n(\%)$ & $73(5)$ & $32(9)$ & $41(4)$ & $0.0010 * * *$ \\
\hline Diabetes duration, years & $12(14)$ & $16(15)$ & $11(13)$ & $<0.0001 * * *$ \\
\hline $\mathrm{HbA}_{1 \mathrm{c}}, \%$ & $7.8 \pm 1.5$ & $7.9 \pm 1.5$ & $7.8 \pm 1.6$ & 0.1841 \\
\hline $\mathrm{HbA}_{1 \mathrm{c}}, \mathrm{mmol} / \mathrm{mol}$ & $62 \pm 12$ & $63 \pm 12$ & $62 \pm 13$ & \\
\hline LDL-cholesterol, mmol/1 & $2.72 \pm 0.96$ & $2.79 \pm 1.01$ & $2.69 \pm 0.93$ & 0.1043 \\
\hline Serum creatinine, $\mu \mathrm{mol} / 1$ & $81(29)$ & $89(38)$ & $79(26)$ & $<0.0001 * * *$ \\
\hline 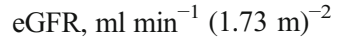 & $76.7 \pm 20.9$ & $68.9 \pm 21.2$ & $79.6 \pm 20.0$ & $<0.0001 * * *$ \\
\hline $\mathrm{uACR}, \mathrm{mg} / \mathrm{mmol}$ & $2.7(9.7)$ & $6.9(31.5)$ & $2.1(6.7)$ & $<0.0001 * * *$ \\
\hline ANGPTL2, ng/ml & $14.8(8.3)$ & $18.1(10.6)$ & $13.9(7.6)$ & $<0.0001 * * *$ \\
\hline NT-proBNP, pg/ml & $102.9(214.3)$ & $240.5(514.4)$ & $77.4(155.8)$ & $<0.0001 * * *$ \\
\hline Soluble TNFR1, pg/ml & $1817(696)$ & $2109(954)$ & $1744(576)$ & $<0.0001 * * *$ \\
\hline Systolic BP, mmHg & $132 \pm 17$ & $136 \pm 19$ & $131 \pm 17$ & $<0.0001 * * *$ \\
\hline Diastolic BP, mmHg & $72 \pm 11$ & $72 \pm 11$ & $72 \pm 11$ & 0.9780 \\
\hline Resting heart rate, bpm & $71 \pm 14$ & $72 \pm 15$ & $71 \pm 13$ & 0.1156 \\
\hline Sinus rhythm, $n(\%)$ & $1281(95)$ & $330(90)$ & $951(97)$ & $<0.0001 * * *$ \\
\hline \multicolumn{5}{|l|}{ Treatment with, $n(\%)$ : } \\
\hline Beta-blockers & $456(34)$ & $135(37)$ & $321(33)$ & 0.1434 \\
\hline ACE inhibitor & $505(37)$ & $170(46)$ & $335(34)$ & $<0.0001 * * *$ \\
\hline ARB & $375(28)$ & $85(23)$ & $290(29)$ & $0.0224 *$ \\
\hline Diuretics & $602(44)$ & $192(52)$ & $410(42)$ & $0.0004 * * *$ \\
\hline Insulin & $799(59)$ & $272(74)$ & $527(53)$ & $<0.0001 * * *$ \\
\hline Aspirin & $377(28)$ & $112(31)$ & $265(27)$ & 0.1841 \\
\hline Statin & $619(46)$ & $162(44)$ & $457(46)$ & $0.04687 *$ \\
\hline
\end{tabular}

Quantitative variables are described as mean $\pm \mathrm{SD}$ or median (interquartile range), unless otherwise specified $* p<0.05, * * * p<0.001$ for those who died vs those who survived

$\mathrm{ARB}$, angiotensin II receptor blocker; uACR, urinary albumin/creatinine ratio
(HR 7.15 [95\% CI 4.19, 12.18]; $p<0.0001$ per log ng/ml). The non-adjusted risks of death and MACE according to serum ANGPTL2 concentrations at inclusion, expressed as a continuous variable, are presented in electronic supplementary material (ESM) Figs 1 and 2, respectively.

In order to identify a clinically meaningful threshold for serum ANGPTL2, the cohort was divided into quartiles (<11.2 [Q1], 11.2-14.7 [Q2], 14.8-19.5 [Q3] and >19.5 [Q4] $\mathrm{ng} / \mathrm{ml}$ ) and the mortality rate was determined for each quartile. While the mortality rate was not significantly different among the first three quartiles (Q1-3), it was significantly higher for patients in Q4 (i.e. those with the highest ANGPTL2 concentrations) (Fig. 1). Next, survival rates (Fig. 2a) and MACE-free survival rates (Fig. 2b) were calculated for each quartile, and significantly worse outcomes were again shown for patients in Q4 as compared with Q1-3. The HR for death in Q4 as compared with Q1 was 3.18 (95\% CI 2.33, 4.34). Of note, patients with ANGPTL2 above $19.5 \mathrm{ng} / \mathrm{ml}$ (Q4) compared with those with ANGPTL2 of $19.5 \mathrm{ng} / \mathrm{ml}$ or less (Q1-3) were older, had more CV risk factors (hypertension) and longer diabetes duration, were more frequently on insulin, and had higher N-terminal pro-B-type natriuretic peptide (NT-proBNP) and TNFR1 levels (although $\mathrm{HbA}_{1 \mathrm{c}}$ levels were comparable), and more patients had a history of stroke and impaired renal function (Table 2). Taken together, a serum ANGPTL2 level of $>19.5 \mathrm{ng} / \mathrm{ml}$ at inclusion was associated with a significantly increased risk of adverse events independent of established $\mathrm{CV}$ or diabetes-associated risk factors and/or markers. 


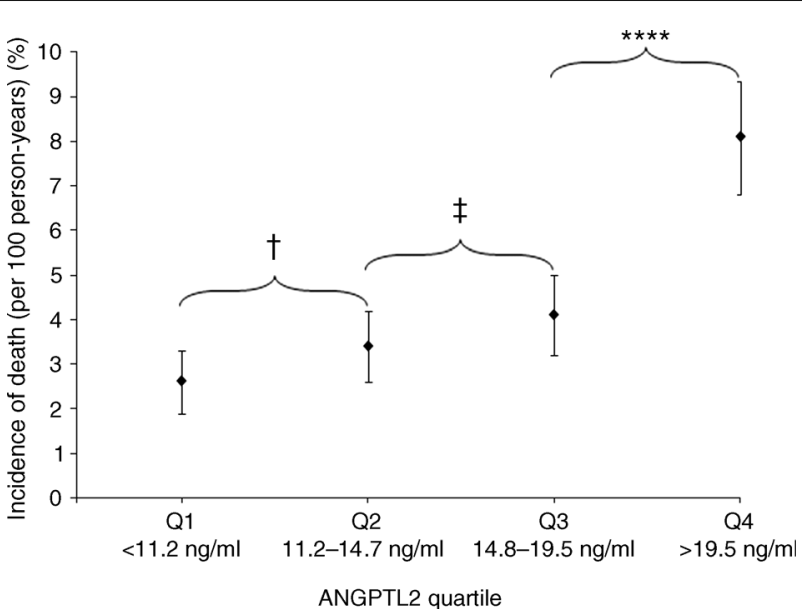

Fig. 1 Incidence of death per 100 person-years according to ANGPTL2 quartiles. Vertical bars show the $95 \%$ CIs. $* * * * p<0.0001 ;{ }^{\dagger} p=0.1470$; ${ }^{\ddagger} p=0.2463$

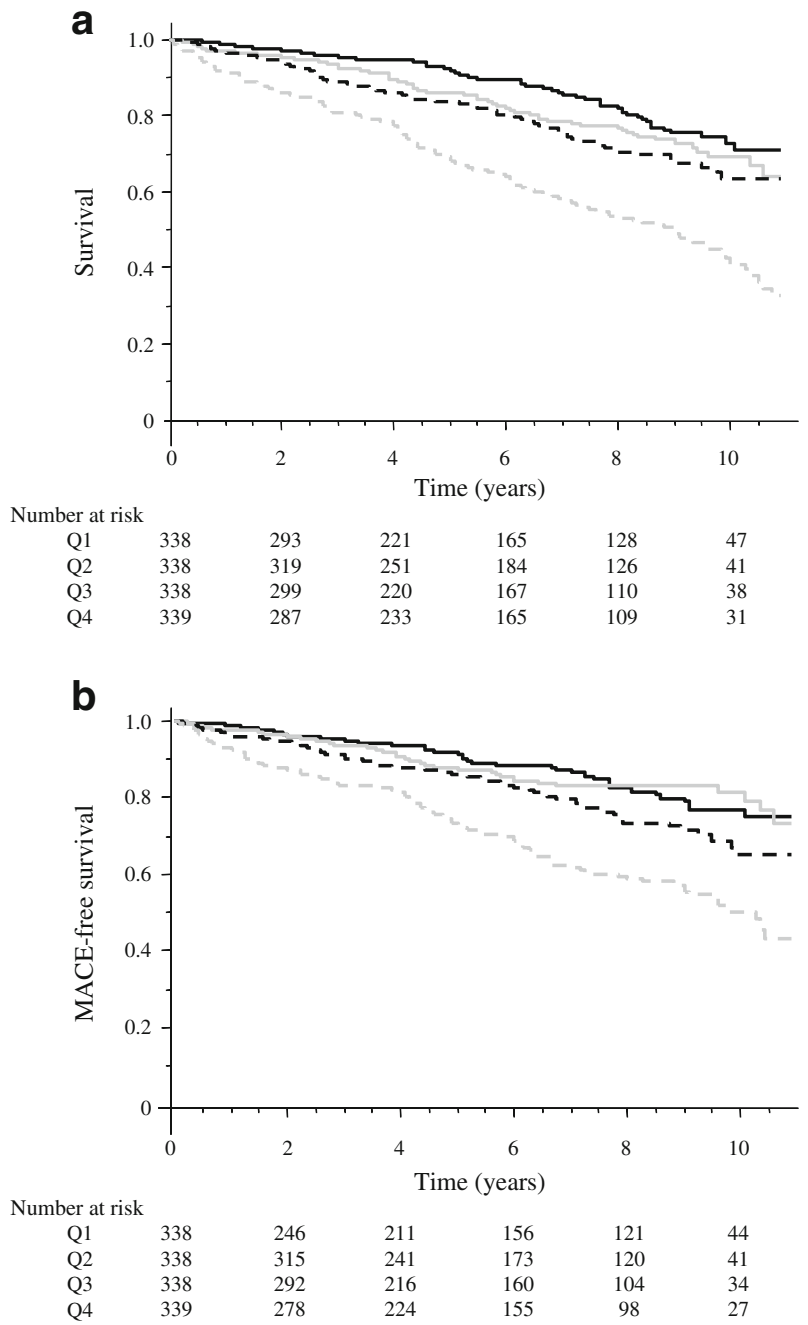

Fig. 2 (a) Survival rates and (b) MACE-free survival rates according to ANGPTL2 quartiles. Black solid line, Q1; grey solid line, Q2; black dashed line, Q3; grey dashed line, Q4
Beyond male sex and advanced age, increased NT-proBNP, history of MI, and ANGPTL2 concentrations remained the variables the most strongly associated both with increased risk of death (Table 3) and MACE (Table 4) in the multivariate analysis after adjustment for confounders. The association between ANGPTL2 concentrations and adverse outcomes remained statistically significant when considering ANGPTL 2 concentrations as a continuous variable.

By using ANGPTL2 concentrations above $19.5 \mathrm{ng} / \mathrm{ml}$ in addition to established prognostic markers in type 2 diabetes mellitus, as presented in Tables 3 and 4, the risk assessment could be significantly improved for death (IDI $0.006 \pm 0.002$, $p=0.0002$ ) and for MACE (IDI $0.007 \pm 0.002 p=0.0014$ ), respectively. Considering another complementary approach with Harrel's C index, the addition of ANGPTL2 concentration in the model non-significantly improved the $\mathrm{C}$ index from 0.766 to 0.767 for death, and from 0.757 to 0.760 for MACE, respectively. The prognostic value of this ANGPTL2 threshold was comparable among different patient subgroups (ESM Fig. 3). We considered the UK Prospective Diabetes Study outcome equation for death in order to evaluate the clinical relevance of ANGPTL2 concentrations in addition to established risk factors, yielding a significant improvement on the prediction of mortality (IDI 0.0012; $p=0.0003$ ).

\section{Discussion}

In this study we have shown, for the first time, a significant association between serum ANGPTL2 concentrations and allcause mortality in type 2 diabetes mellitus patients, reinforcing the concept that ANGPTL2, a proinflammatory and prooxidative factor, may be causal to the development of CVD associated with type 2 diabetes.

Type 2 diabetes patients represent a high-risk population for mortality and CV morbidity, which are driven by macro- and microvascular complications [27, 28]. Despite optimal medical treatment, type 2 diabetes is still associated with a higher risk of more rapid coronary artery disease progression and the development of diabetic cardiomyopathy [29]. CV risk stratification in type 2 diabetes is still essentially based on the assessment of traditional CV risk factors, and the presence of microand macrovascular complications including impaired renal function [30].

Since inflammation plays a key role in the pathophysiology of insulin resistance and diabetes, and because patients with chronic low-grade inflammation are at particularly high risk of adverse events related to atherosclerosis [31], the usefulness of several inflammation and atherosclerosis markers has been evaluated so as to improve $\mathrm{CV}$ risk stratification in patients with type 2 diabetes mellitus $[10,12]$. In the present study, we investigated the prognostic value of the proinflammatory protein ANGPTL2 for risk stratification. The prognostic value of this biomarker, beyond 
Table 2 Comparison of characteristics according to serum ANGPTL2 concentration (Q1-3 vs Q4)

\begin{tabular}{|c|c|c|c|}
\hline Variable & Q1-3 $(n=1014)$ & $\mathrm{Q} 4(n=339)$ & $p$ value \\
\hline ANGPTL2, ng/ml & $12.9(5.7)$ & $25.2(9.7)$ & \\
\hline Men/women, $n(\%)$ & $578(57) / 436(43)$ & $204(60) / 135(40)$ & 0.3055 \\
\hline Age, years & $63 \pm 11$ & $69 \pm 9$ & $<0.0001 * * *$ \\
\hline BMI, $\mathrm{kg} / \mathrm{m}^{2}$ & $31 \pm 6$ & $32 \pm 6$ & 0.1651 \\
\hline Active smoking, $n(\%)$ & $122(12)$ & $26(8)$ & $0.0265^{*}$ \\
\hline Hypertension, $n(\%)$ & $805(79)$ & $307(91)$ & $<0.0001 * * *$ \\
\hline History of myocardial infarction, $n(\%)$ & $141(14)$ & $59(17)$ & 0.1161 \\
\hline History of stroke, $n(\%)$ & $46(5)$ & $27(8)$ & $0.0156^{*}$ \\
\hline Diabetes duration, years & $11(14)$ & $15(15)$ & $<0.0001 * * *$ \\
\hline $\mathrm{HbA}_{1 \mathrm{c}}, \%$ & $7.8 \pm 1.6$ & $7.8 \pm 1.4$ & 0.6996 \\
\hline $\mathrm{HbA}_{1 \mathrm{c}}, \mathrm{mmol} / \mathrm{mol}$ & $61.7 \pm 13$ & $61.7 \pm 11$ & \\
\hline LDL-cholesterol, mmol/1 & $2.77 \pm 0.96$ & $2.72 \pm 0.96$ & 0.2818 \\
\hline Serum creatinine, $\mu \mathrm{mol} / 1$ & $77(24)$ & $98(43)$ & $<0.0001 * * *$ \\
\hline 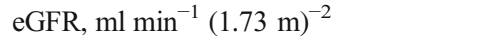 & $81.8 \pm 18.5$ & $61.3 \pm 19.9$ & $<0.0001 * * *$ \\
\hline $\mathrm{uACR}, \mathrm{mg} / \mathrm{mmol}$ & $2.2(6.6)$ & $7.1(29.5)$ & $<0.0001 * * *$ \\
\hline NT-proBNP, pg/ml & $82.0(169.3)$ & $207.2(492.4)$ & $<0.0001 * * *$ \\
\hline TNFR1, pg/ml & $1705(546)$ & $2328(1122)$ & $<0.0001 * * *$ \\
\hline Systolic BP, mmHg & $131 \pm 17$ & $134 \pm 18$ & $0.0075^{* *}$ \\
\hline Diastolic BP, mmHg & $73 \pm 11$ & $72 \pm 12$ & 0.3369 \\
\hline Resting heart rate, bpm & $71 \pm 13$ & $71 \pm 15$ & 0.5793 \\
\hline Sinus rhythm, $n(\%)$ & $972(96)$ & $309(91)$ & $0.0003^{* * *}$ \\
\hline \multicolumn{4}{|l|}{ Treatment with, $n(\%)$ : } \\
\hline Beta-blocker & $320(32)$ & $136(40)$ & $0.0039 * *$ \\
\hline ACE inhibitor & $360(36)$ & $145(43)$ & $0.0166^{*}$ \\
\hline $\mathrm{ARB}$ & $269(27)$ & $106(31)$ & 0.0914 \\
\hline Diuretics & $405(40)$ & $197(58)$ & $<0.0001 * * *$ \\
\hline Insulin & $558(55)$ & $241(71)$ & $<0.0001 * * *$ \\
\hline Aspirin & $278(27)$ & $99(29)$ & 0.5251 \\
\hline Statin & $476(47)$ & $143(42)$ & 0.1278 \\
\hline
\end{tabular}

Quantitative variables are described by mean \pm SD or median (interquartile range), unless otherwise specified ANGPTL2 cut-off level between Q1-3 and Q4: $19.5 \mathrm{ng} / \mathrm{ml}$

$* p<0.05, * * p<0.01, * * * p<0.001$

$\mathrm{ARB}$, angiotensin II receptor blocker; $\mathrm{uACR}$, urinary albumin/creatinine ratio traditional variables, is an important question. The IDI approach suggested that ANGPTL2 serum concentration contributes additional information with regard to mortality risk, although the $\mathrm{C}$ statistics approach was not significant. Prospective studies on this question will help to resolve this issue. Serum ANGPTL2 concentrations were strongly associated not only with CV risk, but also with all-cause mortality. We suggest a threshold for ANGPTL2 serum concentrations of nearby $20 \mathrm{ng} / \mathrm{ml}$, above which the risk of all-cause death increases by $60 \%$.

The upper threshold for normal serum ANGPTL2 concentrations has not been defined. In healthy and physically active volunteers, serum concentrations of ANGPTL2 range from 1 to $3 \mathrm{ng} / \mathrm{ml}$, with similar concentrations between males and females [18]. In the Hisayama study, median serum ANGPTL2 concentrations of 2.7-3.4 ng/ml were reported
[16]; the risk of developing type 2 diabetes was significantly higher in the highest ANGPTL2 quartile $(\geq 3.41 \mathrm{ng} / \mathrm{ml})$. Further studies have reported ANGPTL2 concentrations to average 3.4 vs $2.6 \mathrm{ng} / \mathrm{ml}$ in healthy volunteers [15], while ANGPTL2 concentrations of $4.2 \mathrm{ng} / \mathrm{ml}$ have been found in obese dyslipidaemic women with insulin resistance [21]. In contrast to the small changes in serum ANGPTL2 reported in the literature in relatively healthy patients, we found high concentrations of ANGPTL2 in patients with established chronic diseases, in accordance with a Chinese group [32] that reported very high ANGPTL2 levels in diabetic patients with established kidney disease, ranging from 2.1 to $72.3 \mathrm{ng} / \mathrm{ml}$. It has been suggested that reduced kidney clearance associated with chronic kidney disease could cause an increase in ANGPTL2 levels [33], explaining the discrepancies with 
Table 3 Cox multivariate analysis for the risk of death

\begin{tabular}{|c|c|c|c|c|c|c|}
\hline \multirow[t]{2}{*}{ Variable } & \multicolumn{3}{|c|}{ Maximal model } & \multicolumn{3}{|c|}{ Final model } \\
\hline & $\mathrm{HR}$ & $95 \% \mathrm{CI}$ & $p$ value & HR & $95 \% \mathrm{CI}$ & $p$ value \\
\hline Sex (ref. men) & 0.69 & $0.55,0.87$ & 0.0018 & 0.71 & $0.57,0.89$ & 0.0027 \\
\hline Age (per year) & 1.06 & $1.04,1.07$ & $<0.0001$ & 1.06 & $1.05,1.08$ & $<0.0001$ \\
\hline BMI, $\mathrm{kg} / \mathrm{m}^{2}$ & 1.01 & $0.99,1.02$ & 0.6156 & & & \\
\hline Diabetes duration (per year) & 1.01 & $1.00,1.02$ & 0.0598 & & & \\
\hline History of myocardial infarction & 1.42 & $1.06,1.90$ & 0.0172 & 1.52 & $1.18,1.96$ & 0.0012 \\
\hline Hypertension & 0.93 & $0.61,1.40$ & 0.7091 & & & \\
\hline History of stroke & 1.51 & $1.03,2.21$ & 0.0338 & & & \\
\hline 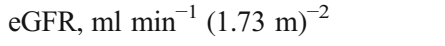 & 1.01 & $1.00,1.02$ & 0.0006 & 1.01 & $1.00,1.02$ & 0.0021 \\
\hline ANGPTL2 (ref. Q1-3) & 1.23 & $0.96,1.58$ & 0.1023 & 1.30 & $1.01,1.66$ & 0.0397 \\
\hline Soluble TNFR1 (per 0.01 log pg/ml) & 1.03 & $1.02,1.04$ & $<0.0001$ & 1.03 & $1.02,1.04$ & $<0.0001$ \\
\hline NT-proBNP (per 100 pg/ml) & 1.01 & $1.00,1.02$ & $<0.0001$ & 1.03 & $1.02,1.04$ & $<0.0001$ \\
\hline Systolic BP, mmHg & 1.00 & $0.99,1.01$ & 0.9276 & & & \\
\hline Sinus rhythm & 0.67 & $0.46,0.97$ & 0.0352 & & & \\
\hline \multicolumn{7}{|l|}{ Treatment with: } \\
\hline Beta-blocker & 1.09 & $0.85,1.41$ & 0.5012 & & & \\
\hline ACE inhibitor & 1.21 & $0.97,1.52$ & 0.0935 & & & \\
\hline Diuretics & 1.13 & $0.90,1.42$ & 0.3017 & & & \\
\hline Insulin & 1.34 & $1.04,1.71$ & 0.0226 & & & \\
\hline Aspirin & 0.80 & $0.62,1.02$ & 0.0686 & & & \\
\hline uACR (ref. <3 mg/mmol) & & & $<0.0001$ & & & $<0.0001$ \\
\hline $3-30 \mathrm{mg} / \mathrm{mmol}$ & 1.36 & $1.06,1.76$ & & 1.44 & $1.13,1.84$ & \\
\hline$>30 \mathrm{mg} / \mathrm{mmol}$ & 2.37 & $1.73,3.24$ & & 2.38 & $1.78,3.17$ & \\
\hline
\end{tabular}

Variables associated with all-cause death at $p<0.10$ in the univariate Cox model were selected for the multivariate 'maximal model'. The 'final model' was determined using multiple backwards stepwise regression analysis applied to the 'maximal model'

uACR, urinary albumin/creatinine ratio other diseases (e.g. coronary heart disease, acute coronary syndrome). As the mechanism of ANGPTL2 clearance is unknown, this remains to be validated. In summary, the higher values measured in this study are most likely due to differences in ethnic background (white), geographical location (France) and/or clinical characteristics (diabetes and kidney disease) compared with previous studies.

Since ANGPTL2 is secreted by adipose tissue, one could expect that increased BMI might be associated with increased serum ANGPTL2 concentrations, as reported in the seminal work by Tabata et al [15]. However, in the present cohort, the fourth quartile of patients, showing the highest ANGPTL2 concentrations and the highest mortality rate, did not have a significantly higher BMI at inclusion than patients in the first three quartiles. In other words, obese type 2 diabetes mellitus patients did not have higher ANGPTL2 concentrations and were not necessarily at higher risk of mortality than nonobese patients. Consequently, an association between obesity on the one hand and increased concentrations of ANGPTL2 and an increased risk of adverse events on the other hand was not obvious in the present cohort.
Rather than the absolute quantity of adipose tissue, the degree of low-grade inflammation, in which adipose tissue is substantially implicated in type 2 diabetes, seems to play a determining role in insulin resistance [34] and, subsequently, for CV complications in these patients [35]. Indeed, inflammation plays a pivotal role in the promotion of vascular damage in type 2 diabetes [36], and the implication of ANGPTL2 as a proinflammatory cytokine favouring macrophage accumulation and activation in this process therefore seems plausible [15]. However, data derived from preclinical research regarding the beneficial [37] or deleterious [15, 34] role of ANGPTL2 in insulin resistance remain somewhat contradictory.

As we described in a previous publication, low-grade inflammation as assessed by soluble TNFR1 concentrations was significantly associated with adverse events in the present cohort [10], thereby confirming and underscoring the link between inflammatory markers and poor outcomes in type 2 diabetes. Interestingly, the additive diagnostic value of serum ANGPTL2 remained highly significant despite adjustment for TNFR1. This finding suggests that ANGPTL2, beyond its role 
Table 4 Cox multivariate analysis for the risk of MACE

\begin{tabular}{|c|c|c|c|c|c|c|}
\hline \multirow[t]{2}{*}{ Variable } & \multicolumn{3}{|c|}{ Maximal model } & \multicolumn{3}{|c|}{ Final model } \\
\hline & HR & $95 \% \mathrm{CI}$ & $p$ value & HR & $95 \% \mathrm{CI}$ & $p$ value \\
\hline Sex (ref. men) & 0.72 & $0.56,0.94$ & 0.0139 & 0.70 & $0.55,0.90$ & 0.0058 \\
\hline Age (per year) & 1.04 & $1.03,1.06$ & $<0.0001$ & 1.04 & $1.03,1.06$ & $<0.0001$ \\
\hline BMI, $\mathrm{kg} / \mathrm{m}^{2}$ & 1.00 & $0.98,1.03$ & 0.7886 & & & \\
\hline Diabetes duration (per year) & 1.01 & $1.00,1.02$ & 0.1821 & & & \\
\hline History of myocardial infarction & 1.74 & $1.27,2.38$ & 0.0006 & 1.73 & $1.32,2.27$ & $<0.0001$ \\
\hline Hypertension & 0.92 & $0.57,1.47$ & 0.7129 & & & \\
\hline History of stroke & 1.52 & $1.00,2.32$ & 0.0508 & & & \\
\hline $\mathrm{HbA}_{1 \mathrm{c}}, \%$ & 1.10 & $1.01,1.20$ & 0.0248 & & & \\
\hline eGFR, $\mathrm{ml} \mathrm{min} \min ^{-1}\left(1.73 \mathrm{~m}^{-2}\right.$ & 1.01 & $1.00,1.02$ & 0.1261 & & & \\
\hline ANGPTL2 (ref. Q1-3) & 1.40 & $1.05,1.85$ & 0.0198 & 1.46 & $1.14,1.88$ & 0.0027 \\
\hline TNFR1 (log pg/ml) & 2.62 & $0.81,8.50$ & 0.1093 & & & \\
\hline NT-proBNP (per 100 pg/ml) & 1.01 & $1.00,1.02$ & $<0.0001$ & 1.03 & $1.02,1.04$ & $<0.0001$ \\
\hline Systolic BP, mmHg & 1.00 & $1.00,1.01$ & 0.4738 & & & \\
\hline Sinus rhythm & 0.68 & $0.45,1.03$ & 0.0679 & & & \\
\hline \multicolumn{7}{|l|}{ Treatment with: } \\
\hline Beta-blocker & 1.03 & $0.77,1.38$ & 0.8267 & & & \\
\hline ACE inhibitor & 1.20 & $0.93,1.55$ & 0.1702 & & & \\
\hline Diuretics & 1.28 & $0.98,1.66$ & 0.0660 & 1.32 & $1.04,1.68$ & 0.0239 \\
\hline Insulin & 1.49 & $1.12,1.98$ & 0.0059 & 1.63 & $1.24,2.14$ & 0.0005 \\
\hline Aspirin & 0.89 & $0.67,1.17$ & 0.3841 & & & \\
\hline uACR (ref. $<3 \mathrm{mg} / \mathrm{mmol}$ ) & & & 0.0004 & & & $<0.0001$ \\
\hline 3-30 mg/mmol & 1.17 & $0.96,1.69$ & & 1.35 & $1.03,1.78$ & \\
\hline$>30 \mathrm{mg} / \mathrm{mmol}$ & 2.04 & $1.43,2.90$ & & 2.29 & $1.68,3.14$ & \\
\hline
\end{tabular}

Variables associated with MACE at $p<0.10$ in the univariate Cox model were selected for the multivariate 'maximal model'. The 'final model' was determined using multiple backwards stepwise regression analysis applied to the 'maximal model'

uACR, urinary albumin/creatinine ratio as a proinflammatory marker, might be related to adverse outcomes by its implication in pathophysiological processes independent of that of TNF. This further supports the concept that ANGPTL2 may be a valuable target in CVD.

Beyond ANGPTL2, three other members of the ANGPTL family have been shown to be associated with CV risk factors and/or CVD. A significant association between ANGPTL3 and atherosclerosis has been reported [38]. The potential of ANGPTL3 inhibition to reduce atherosclerosis is still under evaluation [39]. ANGPTL3 might also be implicated in insulin sensitivity [40] and thereby modulate both lipid and glucose metabolism. ANGPTL4 has also been associated with dyslipidaemia and atherosclerosis in preclinical studies, with interesting data derived from genetic studies [41, 42]. Data concerning the role of ANGPTL8 in diabetes and obesity are conflicting. In mice, ANGPTL8 has been proposed to improve glucose tolerance [43], but increased ANGPTL8 levels in diabetic patients have not been correlated with glucose or insulin levels [44, 45]. Taken together, only ANGPTL4 has been demonstrated to be associated with CV events [46], and none of the ANGPTL family members has been demonstrated to be associated with all-cause death.

Limitations and strengths The major limitation of the present study is that the proposed ANGPTL2 threshold for improving risk stratification in diabetic patients has not yet been confirmed by a validation cohort. Another limitation is that, in the absence of a clinical indication, the majority of patients did not have cardiac imaging at inclusion, which means that the pre-existence of cardiac pathologies such as myocardial hypertrophy or valvular diseases cannot be excluded with certainty. Finally, recruitment in the SURDIAGENE cohort was hospital-based, with a possible recruitment bias for high-risk patients, and might therefore not be representative for an all-comers type 2 diabetes population.

Major strengths of this study are the low dropout rate, the relatively long follow-up, the predefined and clinically highly relevant endpoints, and the robustness of the outcome data in various subgroups. 
Conclusions and perspectives We have shown a strong and independent association of serum ANGPTL2 concentrations with death and MACE in patients with type 2 diabetes mellitus, with or without a history of CV events. Furthermore, we have proposed a clinically meaningful threshold of ANGPTL2, above which the risk of all-cause death is significantly higher. If our results are confirmed, measurement of ANGPTL 2 could become a promising biomarker for improving risk stratification in type 2 diabetes mellitus patients.

Acknowledgements All patients included and followed in the cohort study are warmly thanked for their kind participation in this research. Their GPs are acknowledged for their help in collecting clinical information. M. Thorin is acknowledged for his excellent technical assistance in ANGPTL2 concentrations measurements. J. Arsham (CHU de Poitiers) edited the English of the manuscript.

Centre organisation Recruiting physicians: S. Hadjadj (Coordinator), F. Duengler, L. Labbé, A. Miot, X. Piguel, F. Torremocha, P.-J. Saulnier and R. Maréchaud (CHU de Poitiers, Service Médecine Interne, Endocrinologie et Maladies Métaboliques) Secretarial and technical assistance: all the staff from the Department of EndocrinologyDiabetology (recruitment) and S. Brishoual (CHU de Poitiers, Centre d'Investigation Clinique) and the staff of the Inserm CIC 1402 (biobanking and data management). Centre de Ressources Biologiques of the University of Poitiers (CRB 0033-0068). G. Mauco (Department of Biochemistry, CHU Poitiers, Poitiers, France) and T. Hauet (Inserm U1082, CHU Poitiers, Poitiers, France) are acknowledged for helping in biological determinations.

Adjudication procedure Case inquiry: S. Hadjadj (coordinator), S. Brishoual, C. Divoy, C. Demer, A. Miot, X. Piguel, F. Torremocha, N. Fauvergue (CHU de Poitiers, Service Médecine Interne, Endocrinologie et Maladies Métaboliques), S. Cabasson (CHU de Poitiers, Service Cardiologie), P.-J. Saulnier, P. Sosner, A. A. Kasmi (CHU de Poitiers, Centre d'Investigation Clinique). Local coordination: S. Ragot (coordinator and biostatistician). Adjudication committee: J.-M. Halimi (Chairman, Tours), G. Ducrocq (Paris Bichat), C. Hulin (Poitiers; CHU de Poitiers, Service Cardiologie), P. Llatty (Poitiers) and D. Montaigne (Lille).

Funding The SURDIAGENE cohort was supported by grants from the French Ministry of Health (PHRC-Poitiers 2004; PHRC-IR 2008), the Association Française des Diabétiques (Research Grant 2003) and the Groupement pour l'Etude des Maladies Métaboliques et Systémiques (GEMMS Poitiers, France). ANGPTL2 measurements at the Montreal Heart Institute were supported by the Canadian Institutes of Health Research (MOP 133649 and 14496) and the Foundation of the Montreal Heart Institute.

Duality of interest The authors declare that there is no duality of interest associated with this manuscript. The analysis and interpretation of the data were carried out without the participation of any of the organisations and companies mentioned below.

BG has received speaker's fees from Novartis, Astra Zeneca, Servier and Daiichi Sankyo, and has participated in advisory boards for Novartis.

SH has served as a consultant and/or on advisory panels for Astra Zeneca/Bristol Myers Squibb, has received honorarium or speaking fees from Astra Zeneca/Bristol Myers Squibb, Abbott, Boehringer Ingelheim, Eli-Lilly, Janssen, Merck Sharpe Dome, Novartis, Novo Nordisk, Sanofi, Servier and Takeda, has received research grants from Abbott and Takeda, and has received travel grants from Janssen, Astra Zeneca/ Bristol Myers Squibb, Merck Sharpe \& Dome and Sanofi.

RR has served as a consultant and/or on advisory panels for Astra Zeneca/Bristol Myers Squibb, Novo Nordisk, Sanofi, Merck, Janssen and AbbVie; has received honorarium or speaking fees from Astra Zeneca/
Bristol Myers Squibb, AbbVie, Boehringer Ingelheim, Janssen, Merck, Novartis, Novo Nordisk and Sanofi; has received research grants from Sanofi and Janssen; and has received travel grants from Janssen, Astra Zeneca/Bristol Myers Squib, Sanofi and Janssen.

GD has received speaker's and/or consulting fees from Astra Zeneca, Biotronik, Bristol Myers Squib, Daiichi Sankyo and Lilly, and participated in advisory boards for Lilly.

Contribution statement SH, PS, PJS, SR, PZ, JMH, RR and ET conceived or designed this work. NT-T, MF, SL and SH collected data. BG, PS, EG, GD, DM, PL, VR, NT-T, ET were involved with data analysis and interpretation. BG, NT-T, PS, MF, SL, EG and SH drafted the article. ET, GD, PJS, SR, DM, PL, VR, PZ, JMH and RR critically revised the article. All authors gave final approval of the version to be published. BG is responsible for the integrity of this work as a whole.

\section{References}

1. Turner R, Cull C, Holman R (1996) United Kingdom Prospective Diabetes Study 17: a 9-year update of a randomized, controlled trial on the effect of improved metabolic control on complications in non-insulin-dependent diabetes mellitus. Ann Intern Med 124: 136-145

2. Seshasai SR, Kaptoge S, Thompson A et al (2011) Diabetes mellitus, fasting glucose, and risk of cause-specific death. N Engl J Med 364:829-841

3. Bannister CA, Poole CD, Jenkins-Jones S et al (2014) External validation of the UKPDS risk engine in incident type 2 diabetes: a need for new type 2 diabetes-specific risk equations. Diabetes Care 37:537-545

4. Hayes AJ, Leal J, Gray AM, Holman RR, Clarke PM (2013) UKPDS outcomes model 2: a new version of a model to simulate lifetime health outcomes of patients with type 2 diabetes mellitus using data from the 30 year United Kingdom Prospective Diabetes Study: UKPDS 82. Diabetologia 56:1925-1933

5. van Dieren S, Peelen LM, Nothlings U et al (2011) External validation of the UK Prospective Diabetes Study (UKPDS) risk engine in patients with type 2 diabetes. Diabetologia 54:264-270

6. Berezin AE, Kremzer AA (2013) Circulating osteopontin as a marker of early coronary vascular calcification in type two diabetes mellitus patients with known asymptomatic coronary artery disease. Atherosclerosis 229:475-481

7. Looker HC, Colombo M, Agakov F et al (2015) Protein biomarkers for the prediction of cardiovascular disease in type 2 diabetes. Diabetologia 58:1363-1371

8. Ozturk D, Celik O, Satilmis S et al (2015) Association between serum galectin-3 levels and coronary atherosclerosis and plaque burden/structure in patients with type 2 diabetes mellitus. Coron Artery Dis 26:396-401

9. Price AH, Welsh P, Weir CJ et al (2014) N-terminal pro-brain natriuretic peptide and risk of cardiovascular events in older patients with type 2 diabetes: the Edinburgh Type 2 Diabetes Study. Diabetologia 57:2505-2512

10. Saulnier PJ, Gand E, Ragot S et al (2014) Association of serum concentration of TNFR1 with all-cause mortality in patients with type 2 diabetes and chronic kidney disease: follow-up of the SURDIAGENE Cohort. Diabetes Care 37:1425-1431

11. von Scholten BJ, Reinhard H, Hansen TW et al (2015) Additive prognostic value of plasma $\mathrm{N}$-terminal pro-brain natriuretic peptide and coronary artery calcification for cardiovascular events and 
mortality in asymptomatic patients with type 2 diabetes. Cardiovasc Diabetol 14:59

12. Schottker B, Herder C, Rothenbacher D et al (2013) Proinflammatory cytokines, adiponectin, and increased risk of primary cardiovascular events in diabetic patients with or without renal dysfunction: results from the ESTHER study. Diabetes Care 36:1703-1711

13. Kim I, Moon SO, Koh KN et al (1999) Molecular cloning, expression, and characterization of angiopoietin-related protein. angiopoietin-related protein induces endothelial cell sprouting. J Biol Chem 274:26523-26528

14. Farhat N, Thorin-Trescases N, Mamarbachi M et al (2013) Angiopoietin-like 2 promotes atherogenesis in mice. J Am Heart Assoc 2, e000201

15. Tabata M, Kadomatsu T, Fukuhara S et al (2009) Angiopoietin-like protein 2 promotes chronic adipose tissue inflammation and obesity-related systemic insulin resistance. Cell Metab 10:178-188

16. Doi Y, Ninomiya T, Hirakawa Y et al (2013) Angiopoietin-like protein 2 and risk of type 2 diabetes in a general Japanese population: the Hisayama study. Diabetes Care 36:98-100

17. Endo M, Nakano M, Kadomatsu T et al (2012) Tumor cell-derived angiopoietin-like protein ANGPTL2 is a critical driver of metastasis. Cancer Res 72:1784-1794

18. Thorin-Trescases N, Thorin E (2014) Angiopoietin-like-2: a multifaceted protein with physiological and pathophysiological properties. Expert Rev Mol Med 16, e17

19. Zheng JY, Zou JJ, Wang WZ et al (2011) Tumor necrosis factoralpha increases angiopoietin-like protein 2 gene expression by activating Foxo1 in 3T3-L1 adipocytes. Mol Cell Endocrinol 339: $120-129$

20. Tazume H, Miyata K, Tian Z et al (2012) Macrophage-derived angiopoietin-like protein 2 accelerates development of abdominal aortic aneurysm. Arterioscler Thromb Vasc Biol 32:1400-1409

21. Meng QX, Wen L, Chen XY, Zhong HJ (2013) Association of serum angiopoietin-like protein 2 and epinephrine levels in metabolically healthy but obese individuals: in vitro and in vivo evidence. Exp Ther Med 5:1631-1636

22. Huang CL, Wu YW, Wu CC, Hwang JJ, Yang WS (2015) Serum angiopoietin-like protein 2 concentrations are independently associated with heart failure. PLoS One 10:e0138678

23. Wang Z, Zheng $\mathrm{H}$, Chen $\mathrm{H}$ et al (2015) Elevated serum angiopoietin-like protein 2 in patients with acute coronary syndrome. Arch Med Res 46:257-264

24. Hadjadj S, Fumeron F, Roussel R et al (2008) Prognostic value of the insertion/deletion polymorphism of the ACE gene in type 2 diabetic subjects: results from the Non-insulin-dependent Diabetes, Hypertension, Microalbuminuria or Proteinuria, Cardiovascular Events, and Ramipril (DIABHYCAR), Diabete de type 2, Nephropathie et Genetique (DIAB2NEPHROGENE), and Survie, Diabete de type 2 et Genetique (SURDIAGENE) studies. Diabetes Care 31:1847-1852

25. Levey AS, Stevens LA, Schmid CH et al (2009) A new equation to estimate glomerular filtration rate. Ann Intern Med 150:604-612

26. Hicks KA, Tcheng JE, Bozkurt B et al (2015) 2014 ACC/AHA key data elements and definitions for cardiovascular endpoint events in clinical trials: a report of the American College of Cardiology/ American Heart Association Task Force on Clinical Data Standards (Writing Committee to Develop Cardiovascular Endpoints Data Standards). J Am Coll Cardiol 66:403-469
27. Gu K, Cowie CC, Harris MI (1999) Diabetes and decline in heart disease mortality in US adults. JAMA 281:1291-1297

28. Nathan DM (2015) Diabetes: advances in diagnosis and treatment. JAMA 314:1052-1062

29. Wong TC, Piehler KM, Kang IA et al (2014) Myocardial extracellular volume fraction quantified by cardiovascular magnetic resonance is increased in diabetes and associated with mortality and incident heart failure admission. Eur Heart J 35:657-664

30. Tancredi M, Rosengren A, Svensson AM et al (2015) Excess mortality among persons with type 2 diabetes. N Engl J Med 373:1720 1732

31. de Jager J, Dekker JM, Kooy A et al (2006) Endothelial dysfunction and low-grade inflammation explain much of the excess cardiovascular mortality in individuals with type 2 diabetes: the Hoorn Study. Arterioscler Thromb Vasc Biol 26:1086-1093

32. Li Q, Gong W, Yang Z et al (2013) Serum Angptl2 levels are independently associated with albuminuria in type 2 diabetes. Diabetes Res Clin Pract 100:385-390

33. Usui T, Ninomiya T, Nagata M et al (2013) Angiopoietin-like protein 2 is associated with chronic kidney disease in a general Japanese population: the Hisayama Study. Circ J 77:2311-2317

34. Sasaki Y, Ohta M, Desai D et al (2015) Angiopoietin like protein 2 (ANGPTL2) promotes adipose tissue macrophage and T lymphocyte accumulation and leads to insulin resistance. PLoS One 10, e0131176

35. Berg AH, Scherer PE (2005) Adipose tissue, inflammation, and cardiovascular disease. Circ Res 96:939-949

36. Nilsson J, Bengtsson E, Fredrikson GN, Bjorkbacka H (2008) Inflammation and immunity in diabetic vascular complications. Curr Opin Lipidol 19:519-524

37. Kitazawa M, Nagano M, Masumoto KH, Shigeyoshi Y, Natsume T, Hashimoto S (2011) Angiopoietin-like 2, a circadian gene, improves type 2 diabetes through potentiation of insulin sensitivity in mice adipocytes. Endocrinology 152:2558-2567

38. Hatsuda S, Shoji T, Shinohara K et al (2007) Association between plasma angiopoietin-like protein 3 and arterial wall thickness in healthy subjects. J Vasc Res 44:61-66

39. Dijk W, Kersten S (2016) Regulation of lipid metabolism by angiopoietin-like proteins. Curr Opin Lipidol 27:249-256

40. Robciuc MR, Maranghi M, Lahikainen A et al (2013) Angptl3 deficiency is associated with increased insulin sensitivity, lipoprotein lipase activity, and decreased serum free fatty acids. Arterioscler Thromb Vasc Biol 33:1706-1713

41. Dewey FE, Gusarova V, O'Dushlaine $C$ et al (2016) Inactivating variants in ANGPTL4 and risk of coronary artery disease. N Engl J Med 374:1123-1133

42. Myocardial Infarction G, Investigators CAEC (2016) Coding variation in ANGPTL4, LPL, and SVEP1 and the risk of coronary disease. N Engl J Med 374:1134-1144

43. Yi P, Park JS, Melton DA (2013) Betatrophin: a hormone that controls pancreatic beta cell proliferation. Cell 153:747-758

44. Abu-Farha M, Abubaker J, Al-Khairi I et al (2015) Higher plasma betatrophin/ANGPTL8 level in type 2 diabetes subjects does not correlate with blood glucose or insulin resistance. Sci Rep 5:10949

45. Abu-Farha M, Abubaker J, Noronha F et al (2015) Lack of associations between betatrophin/ANGPTL8 level and C-peptide in type 2 diabetic subjects. Cardiovasc Diabetol 14:112

46. Muendlein A, Saely CH, Leiherer A et al (2014) Angiopoietin-like protein 4 significantly predicts future cardiovascular events in coronary patients. Atherosclerosis 237:632-638 